\title{
Persistent pneumonic consolidations due to secondary organizing pneumonia in a patient recovering from COVID- 19 pneumonia: A case report
}

\section{In-Gyu Bae ( $\nabla$ ttezebae@gmail.com )}

Gyeongsang National University Hospital https://orcid.org/0000-0002-9929-2271

Kyung-Wook Hong

Gyeongsang National University Hospital https://orcid.org/0000-0002-3227-9558

\section{Jung Wook Yang}

Gyeongsang National University Hospital

Kyunglan Moon

Gyeongsang National University Hospital

Jong Duk Kim

Gyeongsang National University Hospital

Sunmi Ju

Gyeongsang National University Hospital

Min-Chul Cho

Gyeongsang National University Hospital

\section{Case Report}

Keywords: COVID-19, SARS-CoV-2, organizing pneumonia, case report

Posted Date: June 23rd, 2020

DOI: https://doi.org/10.21203/rs.3.rs-37580/v1

License: (c) (i) This work is licensed under a Creative Commons Attribution 4.0 International License. Read Full License 


\section{Abstract}

Background: In individuals with coronavirus disease 2019 (COVID-19) pneumonia, radiographic pneumonic infiltrates may persist for several weeks, even after viral clearance, thereby making it difficult to decide on an appropriate treatment and infection control strategy.

Case presentation: We describe a 46-year-old woman with COVID-19 pneumonia who had persistent radiographic pneumonic infiltration and respiratory symptoms for almost 4 weeks after illness onset, despite viral clearance, and was subsequently diagnosed with secondary organizing pneumonia using video-assisted thoracoscopic lung biopsy. Intravenous methylprednisolone was administered at an initial dose of $50 \mathrm{mg} /$ day $(1 \mathrm{mg} / \mathrm{kg})$ for 7 days and was tapered to a dose of prednisolone $30 \mathrm{mg} /$ day following improvement in the patient's respiratory symptoms and chest radiographic findings. The patient was discharged from the hospital 14 days after initiation of corticosteroid treatment. The dose of prednisolone was tapered to $20 \mathrm{mg} /$ day after a month at the outpatient clinic, with further reduction of pneumonic infiltrations seen in a follow-up CT scan on Day 46 of the illness.

Conclusions: To the best of our knowledge, this is the first case report of a pathologically confirmed secondary organizing pneumonia occurring during the convalescent period of COVID-19 pneumonia. Secondary organizing pneumonia should be considered in the differential diagnosis of patients with COVID-19 pneumonia with persistent respiratory symptoms and radiographic pneumonic infiltrations during the recovery phase. In such situations, treatment with steroids may be required, and prolonged isolation of patients with airborne precautions may be unnecessary.

\section{Background}

Coronavirus disease 2019 (COVID-19), caused by severe acute respiratory syndrome coronavirus 2 (SARS-CoV-2) and first identified in Wuhan, China in December 2019, poses a great threat to global public health [1]. As of June 2, 2020, a total of $6,271,577$ cases had been reported in 188 countries with 375,656 deaths [2].

Radiological features frequently observed in patients with COVID-19 pneumonia include bilateral ground-glass opacification (GGO) and consolidation and multilobar involvement with predominantly peripheral or posterior distribution [3, 4]. Furthermore, in COVID-19 pneumonia cases, radiographic pneumonic infiltrates occasionally persist for more than 4 weeks, even after viral clearance. Pan et al. [5] reported that the most severe chest computed tomography (CT) findings were visible on Day 10 after symptom onset, and improvements in imaging findings were observed in $75 \%$ of patients after Day 14.

Here, we report about a patient with COVID-19 who had persistent radiographic pneumonic infiltration and respiratory symptoms until nearly 4 weeks after illness onset, despite repeated negative results for SARS-CoV-2 RNA, no fever, and a normal range of inflammatory markers, thereby making it difficult to decide an appropriate treatment and infection control strategy. This is the first report of COVID-19 pneumonia complicated by secondary organizing pneumonia (OP) during the recovery period, and has implications for treatment and infection control strategies for COVID-19-convalescent patients with persistent respiratory symptoms and radiological abnormalities even after viral clearance.

\section{Case Presentation}

On March 9, 2020, a 46-year-old woman was transferred to our hospital because of dyspnea on exertion, aggravation of pneumonic infiltration, and hypoxia. She had been confirmed to have COVID-19 on March 2, 2020, and had been 
hospitalized in another hospital for 5 days, and lopinavir and ritonavir (LPV/r) treatment (400/100 mg twice daily) was initiated on March 5, 2020. The referring doctor reported that the patient had persistent fever, cough, and a 2-day history of shortness of breath. The patient stated that cough, her first COVID-19 symptom, began on February 27, 2020.

She had a history of hypertension. Her physical examination revealed a body temperature of $36.5^{\circ} \mathrm{C}$, blood pressure of $140 / 90 \mathrm{mmHg}$, pulse of 95 beats/min, respiratory rate of 22 breaths/min, and oxygen saturation of $88 \%$ on room air. The oxygen saturation increased to $95 \%$ with supplemental oxygen of $5 \mathrm{~L} / \mathrm{min}$, delivered via a nasal cannula. Chest high-resolution computed tomography (HRCT) revealed peripheral dominant multifocal consolidation and GGO in both the lungs, which were consistent with pneumonia (Fig. 1A). A nasopharyngeal swab specimen was tested for respiratory viral pathogens using a nucleic acid amplification test, and was positive for parainfluenza virus 3 . The sputum culture had no bacterial growth. Laboratory test results on admission (illness Day 12) showed slightly elevated C-reactive protein levels $(16.6 \mathrm{mg} / \mathrm{L})$, erythrocyte sedimentation rates $(120 \mathrm{~mm} / \mathrm{h})$, lactate dehydrogenase

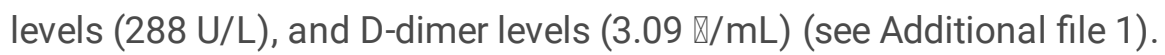

After admission, the patient was administered LPV/r (400/100 mg twice daily) for 14 days (illness Days 8-22), and was administered intravenous nafamostat mesilate $(0.1 \mathrm{mg} / \mathrm{kg} / \mathrm{h})$ for 7 days (illness Days 13-19). Because of the potential of developing combined bacterial pneumonia, ceftriaxone ( $2 \mathrm{~g} /$ day) was administered for 10 days. On Days 1-19 of hospitalization (illness Days 12-30), her vital signs remained stable with no fever, and she did not require oxygen supplementation after hospital Day 12 (illness Day 23) (see Additional file 2). On hospital Day 15 (illness Day 26), follow-up HRCT was performed because she reported pleuritic chest pain and dyspnea on exertion with ongoing cough. Chest radiography revealed slightly increased consolidation in both lungs. HRCT revealed no improvement in the multifocal peripheral dominant consolidations and GGO in either lung, a finding consistent with atypical pneumonia, despite three consecutive negative real-time reverse transcription-polymerase chain reaction (rRT-PCR) results for SARS-CoV-2 RNA (Fig. 1B). Treatment with hydroxychloroquine (400 mg/day) was initiated, and bronchoalveolar lavage (BAL) was performed to evaluate other potential causes of persistent respiratory symptoms and radiographic infiltrates. BAL fluid analysis results were as follows: white blood cell (WBC), 300/区; polymorphonuclear leukocytes (PMN), 4\%; lymphocytes, 21\%; eosinophils, 0\%; and monocytes and macrophages, $75 \%$. The respiratory virus polymerase chain reaction (PCR) multiplex panel, bacterial PCR multiplex panel, and bacterial culture obtained from the BAL fluid revealed no other respiratory pathogens.

On hospital Day 19 (illness Day 30), the patient underwent video-assisted thoracoscopic wedge biopsy of the right upper lung lobe. Microscopic examination revealed intra-alveolar organizing fibroblastic tissues and lymphoplasmacytic infiltration, consistent with OP (Fig. 2A). Additionally, interstitial organization in the peribronchiolar area and peribronchiolar metaplasia was present (Fig. 2B). Gomori methenamine silver and periodic acid-Schiff staining did not reveal any other microorganism. SARS-CoV-2 RNA was not detected in lung tissue using rRT-PCR (Table 1). The patient was thus diagnosed with secondary OP associated with COVID-19 pneumonia. Intravenous methylprednisolone was administered at an initial dose of $50 \mathrm{mg} /$ day $(1 \mathrm{mg} / \mathrm{kg})$ for 7 days and was tapered to a dose of $30 \mathrm{mg}$ of daily prednisolone following improvements in respiratory symptoms and chest radiographic findings. The patient was discharged on hospital Day 37 (illness Day 48) with prednisolone (30 mg/day). The dose of prednisolone was reduced to $20 \mathrm{mg}$ per day at the outpatient clinic after 2 months from admission, since the patient reported no respiratory symptom and follow-up HRCT revealed further gradual improvements in the consolidations and GGO (Fig. 1E).

Table 1 Results of real-time reverse transcriptase polymerase chain reaction testing for severe acute respiratory syndrome coronavirus-2 


\begin{tabular}{|l|c|c|c|c|c|c|c|c|}
\hline & \multicolumn{9}{|c|}{ Day of the test } \\
\hline Sample type & $\begin{array}{c}\text { Feb. 28 } \\
\text { ID 2 }\end{array}$ & $\begin{array}{c}\text { Mar. 11 } \\
\text { ID 14 } \\
\text { HD3 }\end{array}$ & $\begin{array}{c}\text { Mar. 18 } \\
\text { ID 21 } \\
\text { HD 10 }\end{array}$ & $\begin{array}{c}\text { Mar. 20 } \\
\text { ID 23 } \\
\text { HD 12 }\end{array}$ & $\begin{array}{c}\text { Mar. 23 } \\
\text { ID 26 } \\
\text { HD 15 }\end{array}$ & $\begin{array}{c}\text { Mar. 26 } \\
\text { ID 29 } \\
\text { HD 18 }\end{array}$ & $\begin{array}{c}\text { Mar. 27 } \\
\text { ID 30 } \\
\text { HD 19 }\end{array}$ & $\begin{array}{c}\text { Apr. 2 } \\
\text { ID 36 } \\
\text { HD 25 }\end{array}$ \\
\hline $\begin{array}{l}\text { Nasopharyngeal } \\
\text { Oropharyngeal }\end{array}$ & Detected & $\begin{array}{c}\text { Not } \\
\text { detected }\end{array}$ & $\begin{array}{c}\text { Not } \\
\text { detected }\end{array}$ & $\begin{array}{c}\text { Not } \\
\text { detected }\end{array}$ & $\begin{array}{c}\text { Not } \\
\text { detected }\end{array}$ & $\begin{array}{c}\text { Not } \\
\text { detected }\end{array}$ & Not \\
Sputum & Detected & $\begin{array}{c}\text { Detected } \\
\text { (Ct= } \\
\text { (Ct.50) }\end{array}$ & $\begin{array}{c}\text { Not } \\
\text { detected }\end{array}$ & $\begin{array}{c}\text { Not } \\
\text { detected }\end{array}$ & $\begin{array}{c}\text { Not } \\
\text { detected }\end{array}$ & $\begin{array}{c}\text { Not } \\
\text { detected }\end{array}$ & & $\begin{array}{c}\text { Not } \\
\text { detected }\end{array}$ \\
\hline $\begin{array}{l}\text { Bronchoalveolar lavage } \\
\text { fluid }\end{array}$ & & & & & & Not & & \\
\hline Lung tissue & & & & & & & Notected & \\
\hline
\end{tabular}

Abbreviations: Ct, cycle threshold; HD, hospital day; ID, illness day; PCR, polymerase chain reaction.

The patient's nasopharyngeal and oropharyngeal swabs, which were examined on hospital Day 3 (illness Day 14), tested negative for SARS-CoV-2 RNA using rRT-PCR, whereas the sputum remained positive [cycle threshold value of the RNAdependent RNA polymerase gene=31.50]. Nasopharyngeal, oropharyngeal, and sputum specimens obtained on illness Days 21, 23, 26, 29, and 36 tested negative for SARS-CoV-2 RNA. The BAL fluid and lung tissue also tested negative for SARS-CoV-2 RNA on illness Day 29.

\section{Methods}

\section{Specimen collection and nucleic acid extraction}

Sputum and BAL samples were collected without preservatives. Nasopharyngeal and oropharyngeal specimens were collected using flocked swabs and were transported in VTM®: Viral Transport (Copan, Brescia, Italy). Each specimen was processed for RNA extraction using the Real-Prep Viral DNA/RNA kit (Biosewoom, Seoul, Korea) and Real-26 Prep (Biosewoom), which is based on a silica-coated magnetic bead system. Specimens for repeat SARS-CoV-2 RNA testing were collected on illness Days 14, 21, 23, 26, 29, and 36, and included nasopharyngeal and oropharyngeal swabs, sputum, and BAL fluid. SARS-CoV-2 RNA testing was also performed on lung tissue. RNA from a formalin-fixed paraffin-embedded tissue specimen was extracted using the Invitrogen RecoverAll Total Nucleic Acid Isolation kit (Thermo Fisher Scientific, V.A. Graiciuno, Vilnius, Lithuania) and the PureLink RNA Mini Columns (Thermo Fisher Scientific, Carlsbad, CA, USA) according to the manufacturer's protocols.

\section{Real-time reverse transcription polymerase chain reaction amplification}

rRT-PCR amplification was performed using the PowerCheck 2019-nCoV Real-Time PCR kit (Kogenebiotech, Seoul, Korea) and the AB 7500 Fast Real-Time PCR System (Applied Biosystems, Foster City, CA, USA) according to the manufacturer's instructions. The protocols for real-time PCR were as follows: for stage I (reverse transcription), $50^{\circ} \mathrm{C}$ for $30 \mathrm{~min}$; for stage II (denaturation), $95^{\circ} \mathrm{C}$ for $10 \mathrm{~min}$; for stage III, 40 cycles of $95^{\circ} \mathrm{C}$ for $15 \mathrm{~s}$ (denaturation), followed by $60^{\circ} \mathrm{C}$ for $1 \mathrm{~min}$ (primer annealing and extension).

\section{Discussion And Conclusions}

We report a case of COVID-19 pneumonia complicated by the development of secondary OP during the convalescent period. In a study of 99 patients with SARS, which has a 79\% genome sequence homology with SARS-CoV-2, Wong et al.[6] reported that the proportion of patients with GGO and reticulation remained largely unchanged at 3 and 6 months after disease onset [7]. Secondary OP can be caused by infections with viruses such as adenovirus, cytomegalovirus, herpes virus, human immunodeficiency virus, and influenza virus [8] perhaps via immune system 
stimulation by viral antigens [9]. An analysis of the pathological changes in SARS indicated that specimens collected on illness Days 29-46 were characterized by organizing diffuse alveolar damage and fibrosis [10]. Kim et al. [11] previously reported a case of suspected OP after Middle East respiratory syndrome coronavirus infection that was successfully treated with a corticosteroid. Our patient's HRCT findings showed persistent multifocal peripheral dominant consolidations and GGO in both the lungs, even on Day 26 after symptom onset. At that time, she had no fever in the previous 2 weeks, her C-reactive protein levels had normalized, and she had tested negative in three consecutive rRT-PCR assays for SARS-CoV-2 RNA.

Our finding that secondary OP can occur following COVID-19 pneumonia may explain why radiographic infiltrates and respiratory symptoms continue for an extended period after viral clearance and lengthy treatment [5]. In such situations, clinicians need to consider using anti-inflammatory agents such as corticosteroids, which are the mainstay of treatment for OP, and they should avoid inappropriate use of antibiotics or antiviral agents [12]. Spontaneous remission of $\mathrm{OP}$ is rare, and a delay in treating the first episode is a risk factor for relapse $[8,13]$. However, interim guidance from the World Health Organization advises that routine corticosteroids should be avoided in patients with suspected COVID-19 considering the lack of effectiveness and possible harm [14]. Conversely, a previous observational study suggested that methylprednisolone treatment reduced mortality among patients with acute respiratory distress syndrome (ARDS) [15]. However, our patient was administered corticosteroid for treating the secondary OP, not for treating acute SARS-CoV-2 viral pneumonia or ARDS.

Currently, the criteria recommended by the U.S. Centers for Disease Control and Prevention (CDC) and the Korea CDC to discontinue transmission-based precautions include resolution of fever, improvement in respiratory symptoms, and documentation of two consecutive negative results for SARS-CoV-2 RNA at least $24 \mathrm{~h}$ apart [16]. However, it is difficult to determine "the improvement in clinical manifestations" when residual respiratory symptoms and radiographic abnormalities are observed. In addition, false-negative rRT-PCR results for SARS-CoV-2 RNA from throat, nasopharyngeal, or sputum samples are a concern when specimen collection is not performed properly. It is notable that our patient's BAL and lung tissue specimens also tested negative for SARS-CoV-2 RNA, confirming the negative results from nasopharyngeal, oropharyngeal and sputum specimens, despite ongoing respiratory symptoms and pneumonic infiltrations. Our findings suggest that persistent respiratory symptoms and the presence of pneumonic infiltration themselves do not necessarily indicate a need for further isolation of the patient if SARS-CoV-2 molecular tests are negative. These findings imply that negative SARS-CoV-2 rRT-PCR test results may be sufficient for clinicians to decide to release patients from isolation.

Currently, our understanding of the clinical spectrum of COVID-19 is very limited. Although a few studies have described the histopathological findings of lung tissues from patients with COVID-19, most studies conducted postmortem biopsies that revealed diffuse alveolar damage with cellular fibromyxoid exudates, desquamation of pneumocytes, and hyaline membrane formation, indicating ARDS $[17,18]$. However, our case illustrates the pathology of lung tissue during the COVID-19 recovery period when the patient had no fever, no need for supplemental oxygen, normal levels of inflammatory markers, and negative molecular test results for SARS-CoV-2 RNA, despite persistent radiographic pneumonic consolidations. The histopathological findings of our case revealed interstitial organization in the peribronchiolar area and peribronchiolar metaplasia in addition to the common findings of OP. The interstitial organization is likely to have been a reparative change from lung injury caused by SARS-CoV-2 infection. The peribronchiolar metaplasia, which usually occurs because of chronic injury to the terminal airway, might have also been associated with SARS-CoV-2 infection, considering that the case patient was a non-smoker with no history of prior chronic lung injury. 
To the best of our knowledge, this is the first case report of a pathologically confirmed secondary OP occurring during the convalescent period of COVID-19 pneumonia. Based on our findings, secondary OP should be considered in the differential diagnosis of patients with COVID-19 pneumonia with persistent respiratory symptoms and radiographic pneumonic infiltrations during the recovery phase. In such situations, treatment with steroids may be required, and prolonged isolation of patients in a private negative pressure room with airborne precautions may be unnecessary. The incidence of secondary OP developing among convalescent patients with COVID-19 pneumonia is unknown and needs to be clarified in a large scale study.

\section{Abbreviations}

ARDS: acute respiratory distress syndrome; BAL: bronchoalveolar lavage; CDC: Centers for Disease Control and Prevention; COVID-19: coronavirus disease 2019; Ct: cycle threshold; CT: computed tomography; GGO: ground-glass opacification; HD: hospital day; HRCT: high-resolution computed tomography; ID: illness day; LPV/r: lopinavir/ritonavir; OP: organizing pneumonia; PCR: polymerase chain reaction; PMN: polymorphonuclear leukocytes; rRT-PCR: real-time reverse transcription-polymerase chain reaction; SARS-CoV-2: severe acute respiratory syndrome coronavirus 2; WBC: white blood cell.

\section{Declarations}

Ethics approval and consent to participate: This study was approved by the Gyeongsang National University Hospital Institutional Review Board (approval number: 2020-04-009) with a waiver of the requirement for consent because the study had a retrospective design and the data did not contain personal identifiers.

Consent for publication: The patient has provided written informed consent to the publication of this report and the accompanying images.

Availability of data and materials: All data generated or analyzed during this study are included in this published article. This case has not been reported or presented in part or in entirety in any other submission.

Competing interests: The authors declare that they have no competing interests.

Funding: The authors did not receive any funding for the design of the study, collection, analysis, and interpretation of data, or writing the manuscript.

\section{Authors' contributions}

$\mathrm{KH}$ and IB designed the study and wrote the manuscript. JWY performed the histological examination of the lung and contributed to writing the manuscript. KM, JDK, SJ, and MC provided clinical care. All authors contributed significantly to this study and approved the final manuscript.

\section{Acknowledgements}

We thank CK Shim and HJ Lee of the Department of Internal Medicine, Gyeongsang National University Hospital, Jinju, Republic of Korea, for their valuable contributions to this article. We would like to thank Editage for English language editing.

\section{References}


1. Huang C, Wang Y, Li X, Ren L, Zhao J, Hu Y, et al. Clinical features of patients infected with 2019 novel coronavirus in Wuhan, China. Lancet. 2020;395:497-506.

2. Johns Hopkins University CSSE. Coronavirus COVID-19 Global Cases

[https://gisanddata.maps.arcgis.com/apps/opsdashboard/index.html\#/bda7594740fd40299423467b48e9ecf6]. Accessed 2 June 2020.

3. Salehi S, Abedi A, Balakrishnan S, Gholamrezanezhad A. Coronavirus disease 2019 (COVID-19): a systematic review of imaging findings in 919 patients. AJR Am J Roentgenol. 2020. doi: 10.2214/AJR.20.23034.

4. Shi H, Han X, Jiang N, Cao Y, Alwalid O, Gu J, et al. Radiological findings from 81 patients with COVID-19 pneumonia in Wuhan, China: a descriptive study. Lancet Infect Dis. 2020;20:425-34.

5. Pan F, Ye T, Sun P, Gui S, Liang B, Li L, et al. Time course of lung changes at chest CT during recovery from Coronavirus disease 2019 (COVID-19). Radiology. 2020;295:715-21.

6. Wong K-T, Antonio GE, Hui DSC, Ho C, Chan P-N, Ng W-H, et al. Severe acute respiratory syndrome: thin-section computed tomography features, temporal changes, and clinicoradiologic correlation during the convalescent period. J Comput Assist Tomogr. 2004; 28:790-5.

7. Wu A, Peng Y, Huang B, Ding X, Wang X, Niu P, et al. Genome composition and divergence of the novel coronavirus (2019-nCoV) originating in China. Cell Host Microbe. 2020;27:325-8.

8. Drakopanagiotakis F, Polychronopoulos V, Judson MA. Organizing pneumonia. Am J Med Sci. 2008;335:34-9.

9. Schlesinger $\mathrm{C}$, Koss MN. The organizing pneumonias : a critical review of current concepts and treatment. Treat Respir Med. 2006;5:193-206.

10. Cheung OY, Chan JW, Ng CK, Koo CK. The spectrum of pathological changes in severe acute respiratory syndrome (SARS). Histopathology. 2004;45:119-24.

11. Kim I, Lee JE, Kim KH, Lee S, Lee K, Mok JH. Successful treatment of suspected organizing pneumonia in a patient with Middle East respiratory syndrome coronavirus infection: a case report. J Thorac Dis. 2016;8:E1190E1194.

12. Epler GR, Colby TV, McLoud TC, Carrington CB, Gaensler EA. Bronchiolitis obliterans organizing pneumonia. N Engl J Med. 1985;312:152-8.

13. Lazor R, Vandevenne A, Pelletier A, Leclerc P, Court-Fortune I, Cordier JF. Cryptogenic organizing pneumonia. Characteristics of relapses in a series of 48 patients. The Groupe d'Etudes et de Recherche sur les Maladles "Orphelines" Pulmonaires (GERM"O"P). Am J Respir Crit Care Med. 2000;162(2 Pt 1):571-7.

14. WHO. Clinical management of severe acute respiratory infection when COVID-19 is suspected. [https://www.who.int/publications-detail/clinical-management-of-severe-acute-respiratory-infection-when-novelcoronavirus-(ncov)-infection-is-suspected] Accessed 9 April 2020

15. Wu C, Chen X, Cai Y, Xia J, Zhou X, Xu S, et al. Risk factors associated with acute respiratory distress syndrome and death in patients with coronavirus disease 2019 pneumonia in Wuhan, China. JAMA Intern Med. 2020. doi: 10.1001/jamainternmed.2020.0994.

16. Discontinuation of transmission-based precautions and disposition of patients with COVID-19 in healthcare settings (interim guidance) [https://www.cdc.gov/coronavirus/2019-ncov/hcp/disposition-hospitalizedpatients.html] Accessed 22 May 2020

17. Zhang H, Zhou P, Wei Y, Yue H, Wang Y, Hu M, et al. Histopathologic changes and SARS-CoV-2 immunostaining in the lung of a patient with COVID-19. Ann Intern Med. 2020;172:629-32.

18. Xu Z, Shi L, Wang Y, Zhang J, Huang L, Zhang C, et al. Pathological findings of COVID-19 associated with acute respiratory distress syndrome. Lancet Respir Med. 2020;8:420-2. 


\section{Additional Files}

File name and format: Additional file 1.docx

Title of data: Additional Table 1. The patient's serial laboratory test results over the course of hospitalization: March 9 to April 13, 2020

Description of data: This table provides the patient's complete blood count, blood biochemistry, and coagulation test results performed during her hospitalization.

File name and format: Additional file 2.docx

Title of data: Additional Table 2. Disease course by day of illness and days of hospitalization, March 9 to April 12, 2020

Description of data: This table shows the patient's symptoms, treatment and procedures during her hospitalization.

\section{Figures}
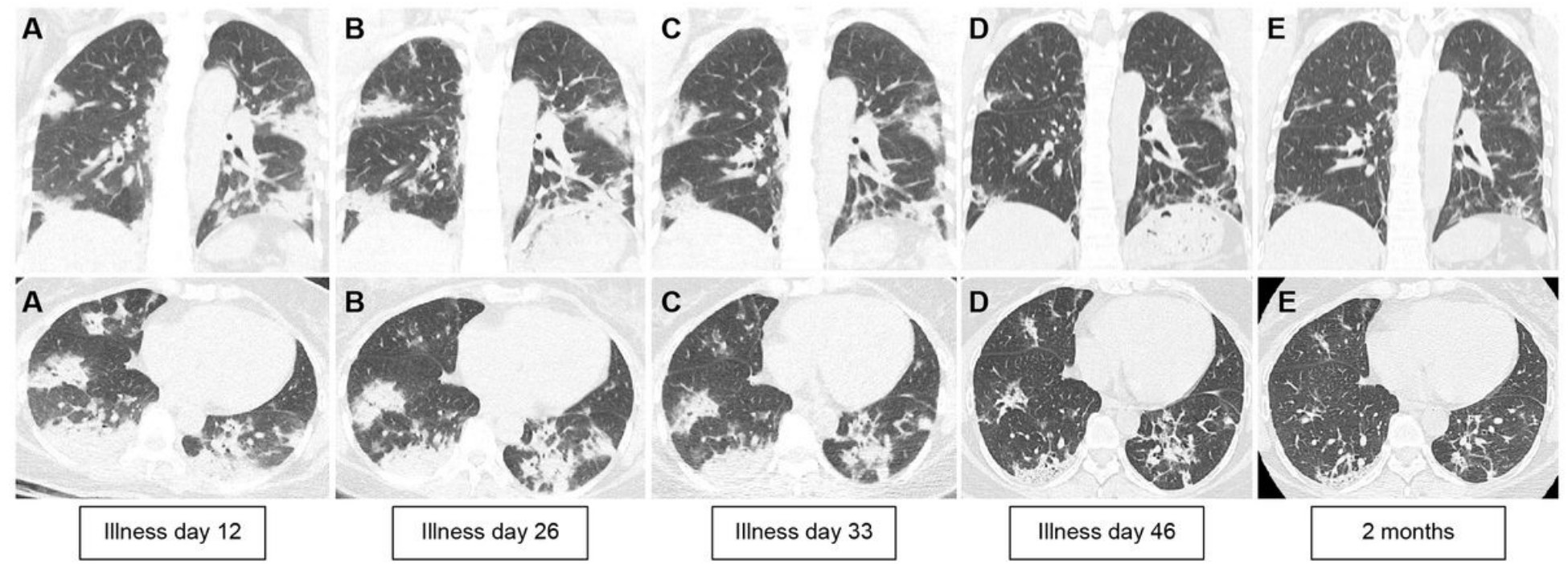

Figure 1

Chest high-resolution computed tomography (HRCT). (A) Chest HRCT on admission (illness Day 12) shows peripheral dominant multifocal consolidation and ground-glass opacification (GGO) in both the lungs. (B) There were no significant changes in bilateral multifocal consolidation and GGO on hospital Day 15 (illness Day 26) or (C) hospital Day 22 (illness Day 33). (D) Follow-up HRCT on hospital Day 35 (illness Day 46) after 12 days of steroid treatment shows slight improvements in multifocal consolidation and GGO in both the lungs. (E) The 2-month follow-up HRCT from admission shows further gradual improvement. 

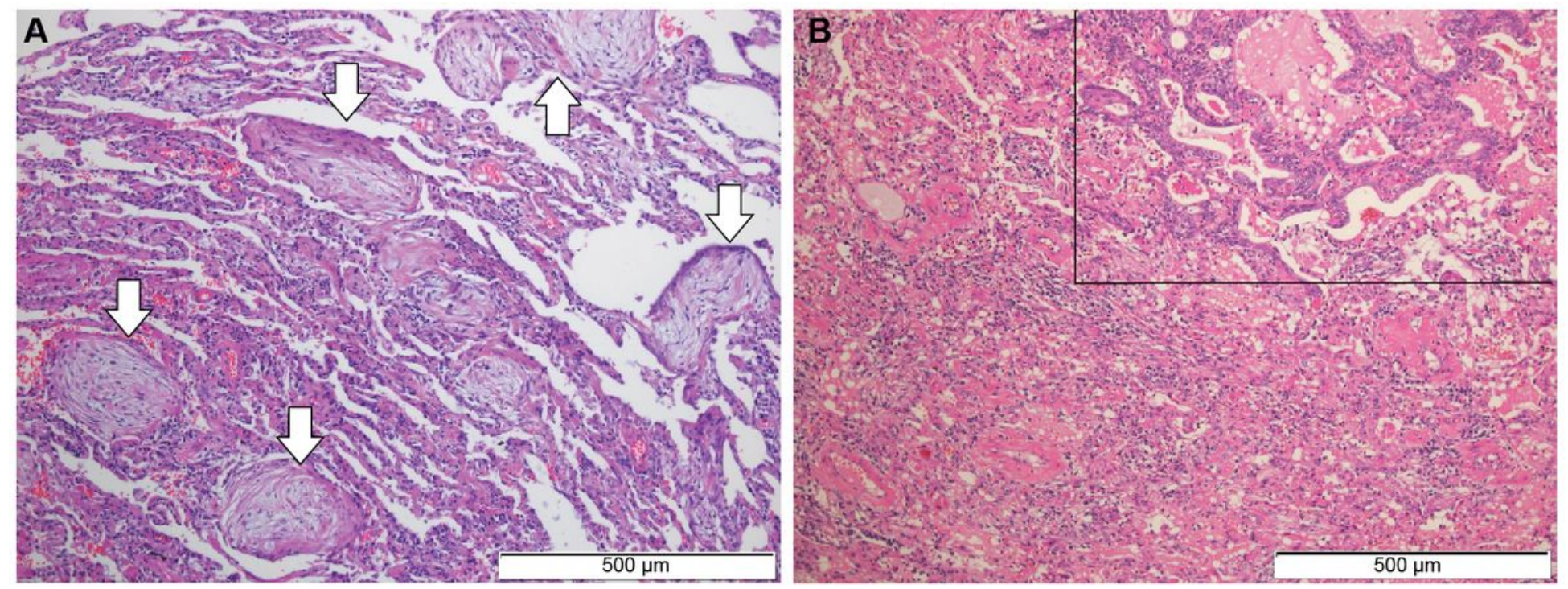

\section{Figure 2}

Histopathologic findings of a wedge resection specimen from the right upper lung lobe. (A) Intra-alveolar organizing fibroblastic tissue (arrows) consistent with organizing pneumonia $(\times 100)$. (B) Interstitial organization and peribronchiolar metaplasia (right upper corner) $(\times 100)$.

\section{Supplementary Files}

This is a list of supplementary files associated with this preprint. Click to download.

- Additionalfile2.docx

- Additionalfile1.docx 